\title{
Aquaporin-2 Promoter Is Synergistically Regulated by Nitric Oxide and Nuclear Factor of Activated T Cells
}

\author{
María F. Albertoni Borghese ${ }^{b}$ Layne M. Bettini ${ }^{a}$ \\ Carlos H. Nitta ${ }^{a}$ Sergio de Frutos ${ }^{a}$ Mónica Majowicz ${ }^{b}$ \\ Laura V. Gonzalez Bosc ${ }^{a}$ \\ a Department of Cell Biology and Physiology, University of New Mexico School of Medicine, \\ Albuquerque, N. Mex., USA; ${ }^{b}$ Biología Celular y Molecular, Facultad de Farmacia y \\ Bioquímica, Universidad de Buenos Aires, Buenos Aires, Argentina
}

\section{Key Words}

AQP2 $\cdot$ MDCK cells $\cdot$ NFATC $\cdot$ Nitric oxide $\cdot$ Nuclear factor $\cdot$ Renal papilla

\begin{abstract}
Background/Aims: We have previously shown that aquaporin-2 (AQP2) is down-regulated in the renal medulla of rats made hypertensive by chronic inhibition of nitric oxide synthase. It has been shown that AQP2 expression is regulated by the calcineurin/nuclear factor of activated T cells (NFATc). Nitric oxide (NO) regulates the activity of NFATc via c-Jun-N-terminal kinase 2 (JNK2). Therefore, we hypothesized that increases in NO enhance NFATc-mediated up-regulation of AQP2 promoter activity. Methods: AQP2 mRNA and protein expression were detected in mouse renal papilla. AQP2 promoter luciferase reporter- and NFAT luciferase reporter-transfected MDCK cells were used to determine AQP2 promoter activity and NFATc activity, respectively. Cells were incubated with classic activators and inhibitors of NFATc and the NO pathway. Results: Our results demonstrate that both $\mathrm{Ca}^{2+}$ and $\mathrm{NO}$ have a synergistic effect resulting in an increase in AQP2 mRNA and protein in mouse papilla and activation of the AQP2 promoter in kidney-derived cells. In addition, NO enhances $\mathrm{Ca}^{2+}$-induced NFATc activation. The underlying mechanism involves increased NFATc nuclear import and decreased export via protein kinase G-mediated inhibition of JNK1/2. Conclusions: This is the first study defining novel regulatory roles for NO and NFATc in the control of AQP2, which is an important renal protein.
\end{abstract}




\section{Introduction}

Water transport through aquaporins (AQPs) is the most important mechanism contributing to kidney water reabsorption [reviewed in ref. 1]. AQP expression correlates with the water permeability of each nephron segment: both the proximal tubules and the descending thin limb of the loops of Henle have high water permeability due to constitutive expression of AQP1. The final checkpoint for renal water reabsorption occurs in the collecting duct, primarily due to the regulated insertion of AQP2 in the apical membrane of principal collecting duct cells. Water permeability in this nephron region is tightly regulated by arginine vasopressin (AVP), which controls AQP2 plasma membrane trafficking and expression [reviewed in ref. 1].

Expression of AQP2 is additionally influenced by urine hypertonicity [2]. Hypertonicity regulates the AQP2 promoter independently of AVP [3]. Water reabsorption increases along the osmotic gradient together with transcription factor tonicity-responsive enhancer binding protein (TonEBP) expression, a member of the nuclear factor of activated T-cell (NFAT) family of transcription factors. This family consists of 5 members, NFAT $1-5$, which share a highly conserved DNA-binding domain [4]. TonEBP (NFAT5), however, differs from the NFATc1-NFATc4 isoforms in two noteworthy ways: (1) it binds DNA as a dimer, whereas NFATc binds DNA as a monomer forming cooperative complexes with Fos and Jun (activator protein 1, AP1), which is activated by protein kinase $\mathrm{C}$ (PKC) [5], and (2) it protects cells from the deleterious effects of high urea by regulating heat shock protein 70 expression and by enhancing AQP2 expression [6,7]. In comparison to the established role of TonEBP in renal physiology and NFATc in the immune, nervous, and cardiovascular systems [8-14], relatively little is known about the role of NFATc in the kidney.

$\mathrm{Li}$ et al. [15] have provided evidence that links $\mathrm{Ca}^{2+}$ signaling and water reabsorption by showing that transcription of AQP2 is enhanced by the calcineurin/NFATc-AP1 pathway. The authors showed that while TonEBP and calcineurin/NFATc-AP1 pathways separately induce AQP2 transcription, a combination of the two pathways enhances AQP2 expression further.

NFATc activation is mediated by a large number of external signals that induce a receptormediated increase in intracellular $\mathrm{Ca}^{2+}$, ensuing activation of the protein phosphatase, calcineurin, which dephosphorylates NFATc leading to its activation and nuclear import [8]. In a previous paper, we have demonstrated that in vascular smooth muscle, nuclear export of NFATc3 has to be down-regulated in order to observe its nuclear accumulation upon calcineurin activation [11]. Via activation of soluble guanylyl cyclase (sGC) and subsequently protein kinase $\mathrm{G}(\mathrm{PKG}), \mathrm{NO}$ is required for inhibiting c-Jun-N-terminal kinase 2 (JNK2, a kinase that phosphorylates, and therefore deactivates, NFATc), leading to reduced NFATc3 nuclear export, while increased intracellular $\mathrm{Ca}^{2+}$ augments NFATc3 nuclear translocation [11]. However, the mechanisms that control NFATc activation have not been explored in kidney cells.

We have previously shown that AQP2 is down-regulated in the outer and inner renal medulla in rats made hypertensive by inhibiting NO synthase (NOS) with chronic administration of L-NAME [16]. Reduced expression of AQP2 in the inner medulla in this chronically NOS-inhibited hypertensive model may be a compensatory mechanism to reduce blood pressure, or it could be due to reduced availability of NO.

The above discussion led us to hypothesize that increases in NO enhance NFATc-mediated up-regulation of AQP2 expression and that this is accomplished by an underlying mechanism involving NO-mediated reduction in NFATc nuclear export by inhibition of JNK1/2. Therefore, reduced NO and NFATc activity could be a compensatory mechanism to alleviate volume expansion in some hypertensive states. Since the roles of NO and NFATc in regulating water balance are highly unexplored, the goal of this study was to define a novel pathway that contributes to the regulation of AQP2, which is an important renal protein. 


\section{Materials and Methods}

All protocols involving animals employed in this study were reviewed and approved by the Institutional Animal Care and Use Committee of the University of New Mexico Health Science Center (Albuquerque, N. Mex., USA) and have been carried out in accordance with EC Directive 86/609/EEC for animal experiments.

\section{Animals}

FVBN male mice (25-30 g) were used. All animals were maintained on a 12-hour lightdark cycle with access to food and water ad libitum. Mice were euthanized with an overdose of pentobarbital (200 mg/kg i.p.) and both kidneys were removed. Kidney papilla was dissected out, incubated in Opti-MEM (Invitrogen) at $37^{\circ} \mathrm{C}$ for $24 \mathrm{~h}$ in the absence or presence of $1 \mu \mathrm{M}$ ionomycin ( $\mathrm{Ca}^{2+}$ ionophore, Calbiochem), $100 \mathrm{nM}$ phorbol 12-myristate 13-acetate (PMA, PKC/AP1 activator, Sigma), $100 \mu \mathrm{M}$ spermine NONOate (NO donor, Calbiochem) or $1 \mu \mathrm{M}$ AVP (dAVP, Sigma), or different combinations of the drugs.

\section{Real-Time PCR}

Total RNA was isolated using the RNeasy mini kit (Qiagen). Total RNA was reverse transcribed to cDNA using a high capacity reverse transcription kit $(\mathrm{A} \& \mathrm{~B})$. For real-time detection of AQP2 transcripts (Mm 00437575_m1) and reference gene ( $\beta$-actin, Mm 00607939 s1), TaqMan gene expression assays (A\&B) were used. The normalized gene expression method $\left(2^{-\Delta \Delta \mathrm{C}_{\mathrm{T}}}\right)$ for relative quantification of gene expression was used [17].

\section{Cell Culture}

Madin-Darby canine kidney (MDCK) cells (from ATCC) [18] were grown in Dulbecco's modified Eagle's medium supplemented with $10 \%$ fetal bovine serum and $1 \%$ penicillin/ streptomycin antibiotic mixture at $37^{\circ} \mathrm{C}$ in $5 \% \mathrm{CO}_{2}$ with controlled humidity.

Cells were treated with ionomycin $(1 \mu \mathrm{M})$ and PMA (100 nM; Io+PMA in the presence, absence, or different combinations of cyclosporine A (CsA, $1 \mu \mathrm{M}$, Calbiochem), NO donor $(100 \mu \mathrm{M})$, sGC inhibitor $1 \mathrm{H}-[1,2,4]$ oxadiazolo-[4,3-a]quinoxalin-1-one (ODQ, $10 \mu \mathrm{M}$, Calbiochem), and PKG inhibitor Rp-8-pCPT-cyclic guanosine monophosphate (cGMP), and PKGi (10 $\mu \mathrm{M}$, Calbiochem). Leptomycin B (40 nM, Sigma) was used to inhibit NFATc3 nuclear export. For the promoter studies, cells were treated for $24 \mathrm{~h}$. For the import/export studies, treatment did not last $>1 \mathrm{~h}$.

\section{Plasmids}

9xNFATc luciferase reporter plasmid was kindly provided by Dr. Molkentin (Department of Pediatrics, Children's Hospital Medical Center, Cincinnati, Ohio, USA). The 9xNFATc luciferase plasmid has 9 NFATc binding sites upstream of the luciferase gene.

AQP2 promoter luciferase reporter plasmid was kindly provided by Dr. Chen (Department of Internal Medicine/Renal Division, Washington University School of Medicine, St. Louis, Mo., USA). The AQP2 promoter reporter construct contains a 600-bp DNA fragment from the mouse AQP2 proximal promoter (nucleotides 8923-9522, GenBank AY055468) cloned in a luciferase vector (pGL3-basic vector, Promega). Six NFAT binding sites were previously identified by Li et al. [15] in the AQP2 promoter. Therefore, we also tested mutants pAQP2-MT3, pAQP2-MT2, and pAQP2-MT1 in which the putative NFATc sites were mutated by PCR-directed mutagenesis [15]. These plasmids were also kindly provided by Dr. Chen. pGL3-basic empty vector was used as control. TK-renilla reporter plasmid (Promega) was used to normalize for differences in transfection efficiencies. 
NFATc3-enhanced green fluorescent protein (EGFP) expression vector was created by Dr. F. McKeon (Harvard University, Cambridge, Mass., USA) and kindly provided by Dr. L.F. Santana (Washington State University, Seattle, Wash., USA).

Transient Transfections and Luciferase Assay

MDCK cells were transfected with the appropriate combination of vectors using Lipofectamine 2000 reagent (Invitrogen) or Nucleofector (Lonza). Luciferase and renilla activity were simultaneously detected using a dual-luciferase reporter assay system (Promega). Renilla activity was used to normalize luciferase activity.

\section{Fluorescence Microscopy}

NFATc3-EGFP-transfected MDCK cells were seeded on microscope coverslips and cultured for at least $24 \mathrm{~h}$. At the time of experimentation, a coverslip was mounted on a heating plate chamber and maintained in Opti-MEM at $\sim 37^{\circ} \mathrm{C}$ during the experiments.

Nuclear EGFP fluorescence was monitored using a Nikon Diaphot 300 at a magnification of $\times 200$. Individual MDCK cells were imaged once every minute for $30 \mathrm{~min}$. Images were captured using Andor IQ 1.9 software. Nuclear fluorescence (F) was background corrected and expressed as $\mathrm{x}$-fold change from baseline nuclear fluorescence $\left(\mathrm{F} / \mathrm{F}_{0}\right.$; Metamorph Universal Imaging software).

AQP2 was detected by immunofluorescence microscopy in sections of mouse kidney papilla. After $24 \mathrm{~h}$ of incubation with the treatments described in the animals section, the papilla was fixed in $4 \%$ formaldehyde in PBS, cryoprotected with $30 \%$ sucrose in PBS and embedded in OCT. Sections were permeabilized, blocked, and stained with rabbit antiAQP2 (1/200, Abcam) followed by anti-rabbit Cy5 (Jackson ImmunoResearch Laboratories). Nuclei were stained with SYTOX Green (Invitrogen). Sections were examined using a $\times 40$ objective on an EVOS fluorescence microscope (AMG). The same fluorescence threshold was applied to all the images, and average intensity was determined using Metamorph software (Universal Imaging). Specificity of immune staining was confirmed by the absence of fluorescence in tissues incubated with primary or secondary antibodies alone.

\section{Detection of p-JNK1/2 Levels by Western Blot}

MDCK cells were lysed using RIPA buffer (Pierce) at $4^{\circ} \mathrm{C}$ and centrifuged at $16,000 \mathrm{~g}$ for 2 min. Protein concentration was determined in the supernatant using a BCA protein assay kit (Pierce). Supernatants (20 $\mu \mathrm{g} / \mathrm{lane})$ were resolved by SDS-PAGE and proteins transferred to PVDF membranes. The membranes were blocked with blocking buffer from Odyssey (LICOR Biosciences). The membranes were incubated with primary antibodies, rabbit anti-pJNK1/2 (pT183/pY185; Invitrogen) $1 / 100$ and mouse anti- $\beta$-actin (Sigma) $1 / 5,000$, in $0.1 \%$ Tween PBS-buffered saline solution at $4^{\circ} \mathrm{C}$ overnight, washed, and incubated for $1 \mathrm{~h}$ with 1/10,000 goat anti-rabbit IRdye $800 \mathrm{CW}$ and goat anti-mouse IRdye 680 (LI-COR Biosciences). The membrane was scanned using the Odyssey infrared imaging system (LI-COR Biosciences). The results were expressed as the ratio between $\mathrm{p}$-JNK1/2 over $\beta$-actin fluorescence intensity and normalized to control.

\section{Statistics}

Results are expressed as the mean \pm SEM of at least 4 experiments. Statistical significance was tested at the $95 \%(\mathrm{p}<0.05)$ confidence level using one- or two-way repeated-measure ANOVA. NFATc3-EGFP nuclear import and export curves were obtained by non-linear regression fit to a one-phase association equation and one-phase exponential decay equation, respectively. 

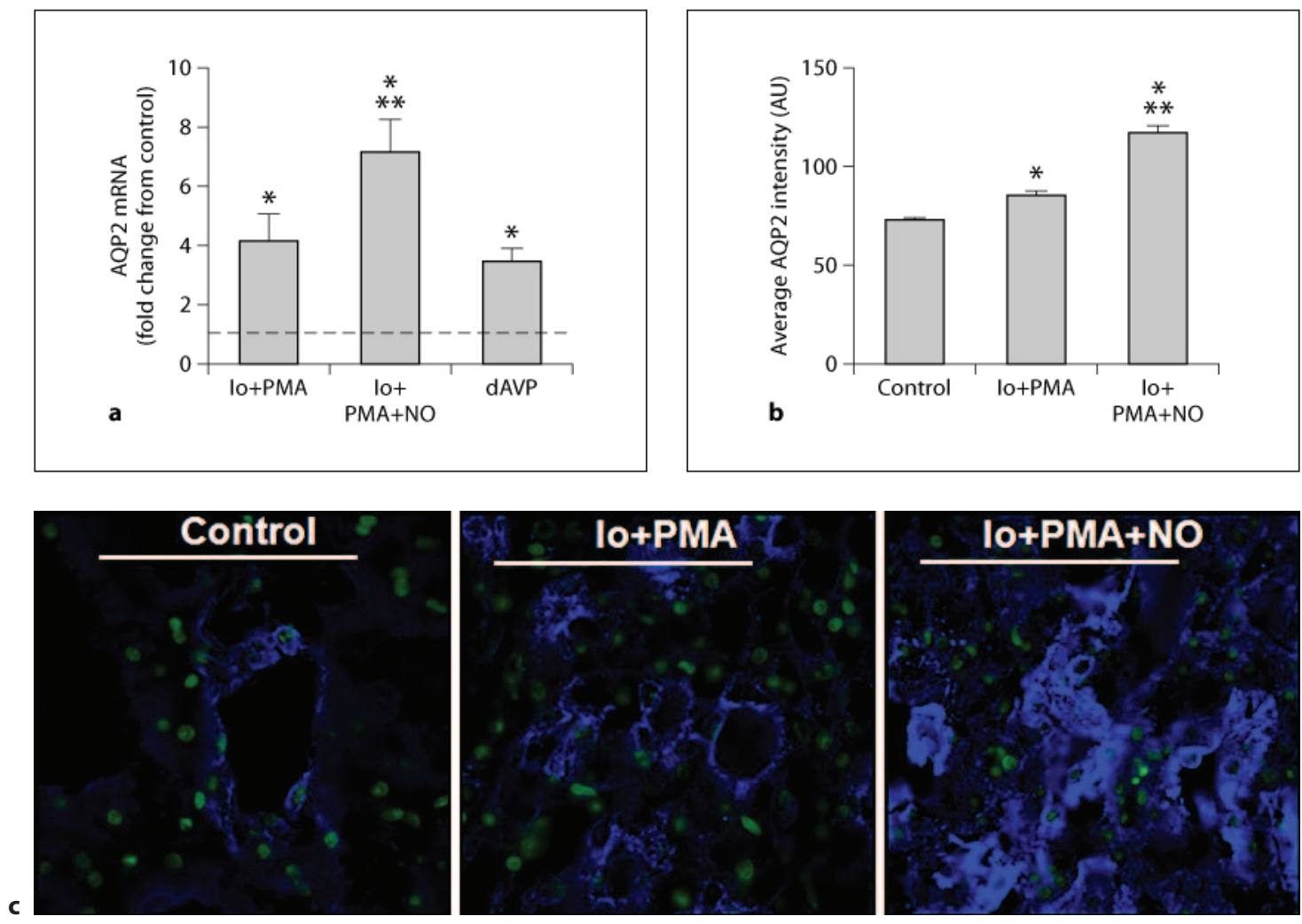

Fig. 1. $\mathrm{NO}$ enhances $\mathrm{Ca}^{2+} / \mathrm{PKC}$-induced increases in AQP2 mRNA and protein expression in mouse renal papilla. Renal papilla was isolated from mice and treated for $24 \mathrm{~h}$ with vehicle, ionomycin (1 $\mu \mathrm{M})$, PMA (100 nM), NO donor $(100 \mu \mathrm{M})$, or dAVP $(1 \mu \mathrm{M}$, positive control) in different combinations. a AQP2 mRNA was detected by real-time PCR (TaqMan assay). b Effect of Io+PMA and Io+PMA+NO on AQP2 protein expression assessed by immunofluorescence. ${ }^{*} \mathrm{p}<0.05$ vs. control; ${ }^{*} \mathrm{p}<0.05$ vs. Io+PMA. $\mathrm{n}=6$ for mRNA and $n=3$ for protein. $\mathbf{c}$ Representative images of AQP2 immunofluorescence staining of collecting duct cells of the renal papilla. Scale bar $=100 \mu \mathrm{m}$.

\section{Results}

NO Enhances $\mathrm{Ca}^{2+} / P K C$-Induced Increase in AQP2 mRNA and Protein in Mouse Renal Papilla

We have shown that AQP2 is down-regulated in the outer and inner renal medulla in rats made hypertensive by chronic inhibition of NOS [16]. NFATc has been shown to regulate AQP2 expression in $\mathrm{mpkCCD}_{\mathrm{c14}}$ cells (immortalized murine principal collecting duct cells) [15]. Elevations in intracellular $\mathrm{Ca}^{2+}$ activate NFATc and activation of PKC increases AP1 activity/NFATc co-factor [reviewed in ref. 8]. Therefore, we tested whether the $\mathrm{Ca}^{2+} / \mathrm{PKC} /$ NFATc/AP1 pathway regulates AQP2 expression in mouse kidney papilla. This region of the kidney was used because it is rich in collecting ducts, and AQP2 is primarily expressed at the apical membrane of principal collecting duct cells [reviewed in ref. 1]. Isolated papilla from mouse kidney was cultured for $24 \mathrm{~h}$ in the absence or presence of the calcineurin/NFATc activator ionomycin and the PKC/AP1 activator PMA, and mRNA and protein levels were measured. dAVP was used as positive control. In addition, we tested whether NO can modulate any effect of Io+PMA. Figure la shows that indeed Io+PMA significantly up-regulated AQP2 mRNA expression. Co-treatment with NO significantly enhanced Io+PMA-induced 
Fig. 2. a Calcineurin/NFATc regulates AQP2 promoter. Experiments performed in AQP2 promoter luciferase reporter-transfected MDCK cells. b Calcineurin/NFATc pathway is transcriptionally active in MDCK cells. Experiments performed in NFAT luciferase reporter-transfected MDCK cells. a, b Cells were treated for $24 \mathrm{~h}$ with vehicle, ionomycin (1 $\mu \mathrm{M})$, PMA (100 $\mathrm{nM})$ and CsA $(1 \mu \mathrm{M})$, or their combination. * $\mathrm{p}<0.05$ vs. control $(\mathrm{n}=6)$. $\mathrm{RLU}=$ Relative luciferase units.
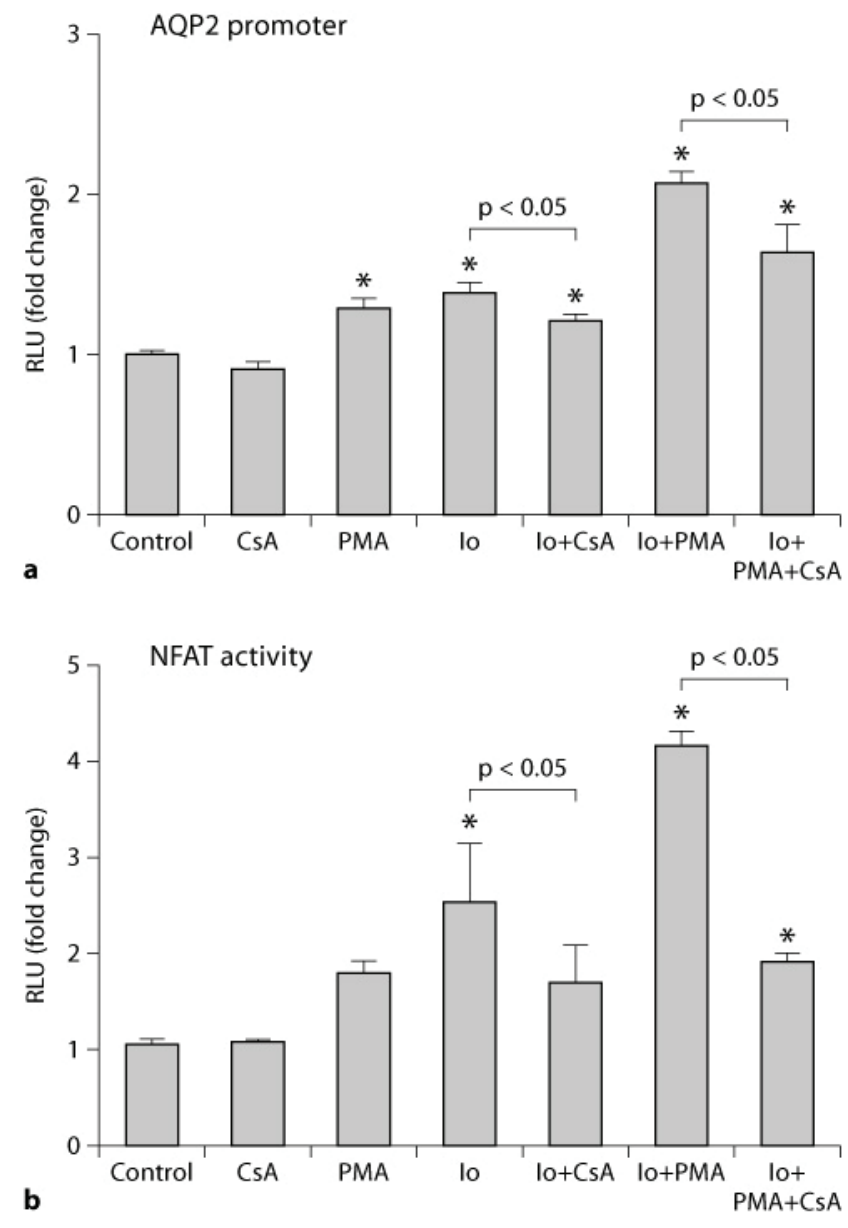

increases in AQP2 mRNA expression. Figure $1 \mathrm{~b}$ and $1 \mathrm{c}$ show that $\mathrm{NO}$ also significantly enhanced Io+PMA-induced increases in AQP2 fluorescence staining in collecting duct cells of the renal papilla.

\section{Regulation of AQP2 Promoter by NO and NFATc in MDCK Cells}

In order to explore the mechanism of NO-enhanced Io+PMA-induced up-regulation of AQP2 expression, we studied the regulation of AQP2 promoter by NFATc and NO in cultured MDCK cells.

Li et al. [15] have shown that activation of NFATc and AP1 increases AQP2 promoter activity in mpkCCD $\mathrm{cl}_{4}$ cells. We have obtained similar results in MDCK cells transfected with the same AQP2 promoter luciferase reporter. As expected, AQP2 promoter activity increased in cells treated with Io+PMA (fig. 2a). This activation was attenuated by the calcineurin inhibitor, CsA. In addition, the same treatments were applied to cells transfected with an NFATc reporter confirming that they also increase NFATc activity in these cells (fig. 2b). These treatments had no effect on cells transfected with empty reporter plasmid (data not shown).

Consistent with our hypothesis, an NO donor had a synergic effect with Io+PMA on AQP2 promoter activity (fig. 3a) suggesting that NO enhances NFATc activity. NO by itself 
Fig. 3. a $\mathrm{NO}$ enhances NFATc/ AP1-mediated AQP2 promoter activity. Experiments performed in AQP2 promoter luciferase reporter-transfected MDCK cells. b NO enhances NFATc activity. Experiments performed in NFAT luciferase reporter-transfected MDCK cells. a, b Cells were treated for $24 \mathrm{~h}$ with vehicle, ionomycin (1 $\mu \mathrm{M})$, PMA (100 nM), CsA $(1 \mu \mathrm{M})$, NO donor $(100 \mu \mathrm{M}), \mathrm{ODQ}$ $(10 \mu \mathrm{M})$, or the combination of them. ${ }^{*} \mathrm{p}<0.05$ vs. control. ** $\mathrm{p}<0.05$ vs. Io+NO+PMA ( $\mathrm{n}=$ 6). RLU = Relative luciferase units.
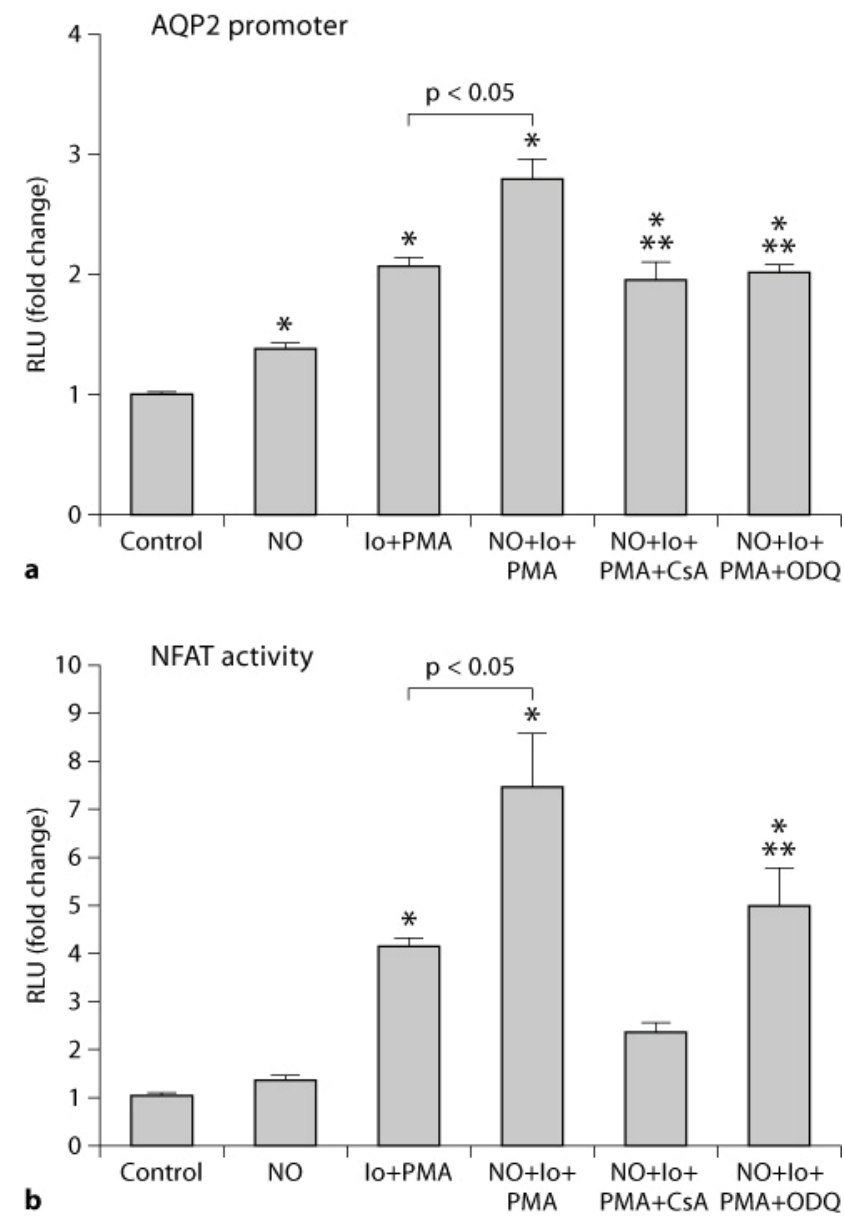

caused a significant, albeit small, increase in AQP2 promoter activity. NO also enhanced Io+PMA-induced increases in NFATc activity (fig. 3b). Treatment with NO only had no effect.

The role of calcineurin in the synergistic effect of NO and NFATc both in AQP2 promoter and NFATc activity was established by inhibiting calcineurin with CsA. CsA attenuated NO-enhanced NFATc-mediated AQP2 promoter activation (fig. 3a). CsA reduced luciferase activity almost to the level of NO treatment alone. Furthermore, CsA prevented NOenhanced Io+PMA-induced NFATc activation (fig. 3b).

In order to determine if $\mathrm{NO}$ acts via the previously described cGMP/PKG pathway, cells were incubated with an sGC inhibitor, ODQ. ODQ significantly reduced both NO-enhanced Io+PMA-induced AQP2 promoter and NFATc activity (fig. 3).

NFAT Sites within the AQP2 Proximal Promoter Mediate the Regulation of AQP2

Expression in Response to Calcium and NO

To further determine whether the synergistic effect of $\mathrm{NO}$ and $\mathrm{Ca}^{2+} / \mathrm{PKC} / \mathrm{AP} 1$ signaling is dependent on the demonstrated NFAT binding sites [15], MDCK cells were transfected with pAQP2-MT1, pAQP2-MT2, and pAQP2-MT3 constructs that have the first, the last 5, or all 6 NFAT binding sites mutated within the proximal AQP2 promoter. As previously 
Fig. 4. Single nucleotide mutations in 6 NFATc binding sites in the AQP2 promoter prevent NFATc-mediated AQP2 promoter activation and $\mathrm{NO}$ effect. MDCK cells were transfected with WT AQP2 promoter luciferase reporter and mutated constructs MT1, MT2 and MT3 as depicted in the upper graph. Cells were treated for $24 \mathrm{~h}$ with vehicle, ionomycin (1 $\mu \mathrm{M})$, PMA $(100 \mathrm{nM})$, and NO donor $(100 \mu \mathrm{M})$ in different combinations. ${ }^{*} \mathrm{p}<$ 0.05 vs. control; ${ }^{* *} \mathrm{p}<0.05$ vs. Io+PMA within group; ${ }^{* * *} \mathrm{p}<$ 0.05 vs. Io+PMA WT; ${ }^{* * * *} \mathrm{p}<$ 0.05 vs. Io+PMA+NO WT $(\mathrm{n}=$ 6). $\mathrm{RLU}=$ Relative luciferase units.
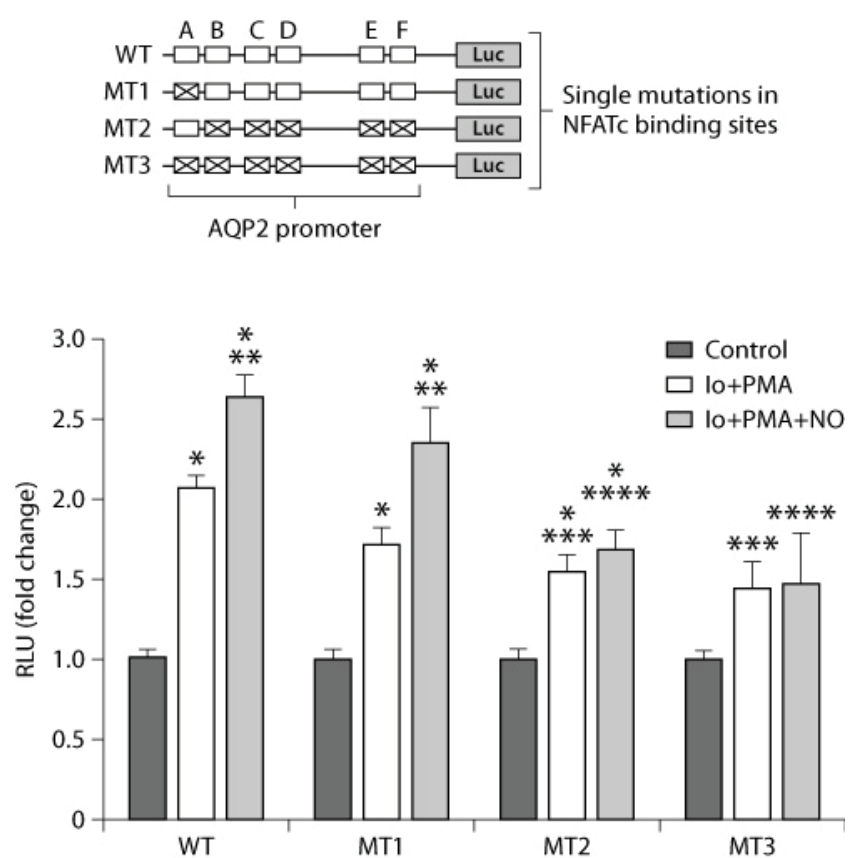

shown by Li et al. [15], the induction by Io+PMA was completely lost in pAQP2-MT3 with all 6 sites mutated, confirming the importance of these sites in the induction of AQP2 promoter (fig. 4). No significant difference was detected in AQP2 promoter activity after stimulation with Io+PMA in pAQP2-MT3-expressing cells. The synergistic effect of NO was also absent. In addition, the mutation of 5 sites in PAQP2-MT2 reduced the response to both Io+PMA and NO+Io+PMA (fig. 4). There was no difference in the response to both Io+PMA and $\mathrm{NO}+\mathrm{Io}+\mathrm{PMA}$ between pAQP2 wild type (WT) and pAQP2-MT1, suggesting the first NFAT binding site is not enough to regulate AQP2 promoter activity. We did not observe any significant difference in baseline (control) luciferase expression between AQP2 WT and mutant-expressing cells (pAQP2-WT $=1.80 \pm 0.11$, pAQP2-MT1 $=1.71 \pm 0.15$, pAQP2$\mathrm{MT} 2=1.40 \pm 0.09, \mathrm{pAQP} 2-\mathrm{MT} 3=1.65 \pm 0.13$ relative luciferase units) suggesting NFATc binding sites are not required for basal AQP2 promoter activity.

\section{NO Affects NFATc Nuclear Accumulation}

In order to further explore how NO modulates NFATc transcriptional activity, the effect of NO on NFATc nuclear import/export was determined.

To study NFATc nuclear export, the transcription factor must be localized in the nucleus at the beginning of the experiment, so NFATc3-EGFP-transfected cells were treated with ionomycin for $1 \mathrm{~h}$ prior to commencing the experiment. CsA was then added in the presence or absence of NO. The translocation was imaged and analyzed, as described in Materials and Methods. As expected, NO significantly attenuated NFATc3-EGFP nuclear export, as shown in the representative images and kinetic curves (fig. $5 \mathrm{a}$ and b, respectively). NO significantly increased the plateau of NFATc3 nuclear export (fig. 6b).

In order to determine if $\mathrm{NO}$ acts via the previously described cGMP/PKG pathway, NFATc3-EGFP-transfected cells were treated with CsA and NO and a selective PKG inhibitor (PKGi). Indeed, PKG inhibition prevented NO-induced reduction in NFATc nuclear ex- 
Fig. 5. NO decreases NFATc3EGFP nuclear export. MDCK cells transfected with NFATc3EGFP plasmid were pretreated with ionomycin $(1 \mu \mathrm{M})$ for $1 \mathrm{~h}$ to induce maximum nuclear accumulation of NFATc3, then CsA $(1 \mu \mathrm{M})$ was added (time 0$)$ to inhibit further NFATc3 nuclear import; cells were treated with vehicle (control), NO donor (100 $\mu \mathrm{M})$, and PKGi (10 $\mu \mathrm{M})$, and imaged for $30 \mathrm{~min}$. a Representative images of control and NO-treated cells. b Kinetic curve of NFATc3-GFP nuclear export. Fluorescence was expressed as $\mathrm{F} / \mathrm{F}_{0}\left(\mathrm{~F}_{0}=\right.$ fluorescence at baseline). ${ }^{*} \mathrm{p}<0.05$ from 28 to $30 \mathrm{~min}$ between $\mathrm{NO}$ and control and $\mathrm{NO}+\mathrm{PKGi}(10 \mu \mathrm{M})$. No significant difference is noted between control and NO+PKGi. Right panel shows a blow-up of the initial rate of export.

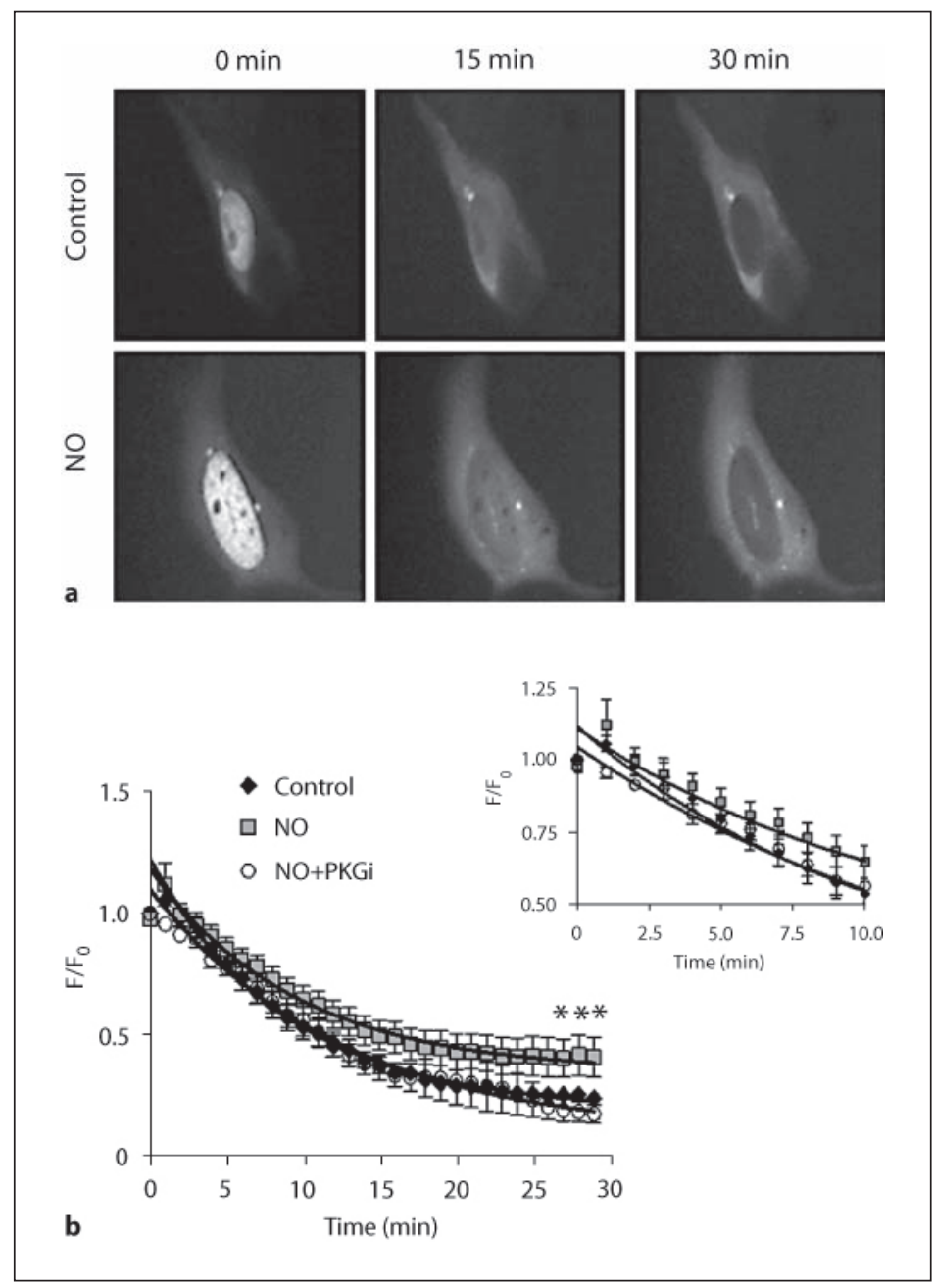

port (fig. 5b). PKGi significantly decreased the rate of NFATc3 nuclear export and the plateau (fig. 6b).

It is known that phosphorylation by several serine/threonine kinases such as GSK3 $\beta$, calmodulin (CaM) kinase II and JNK1/2 regulate NFATc nuclear export [reviewed in ref. 10]. Therefore, we determined whether inhibition of these kinases would mimic the effect of NO on NFATc nuclear export. NFATc3-EGFP-transfected cells were treated with CsA and JNK1/2 inhibitor (JNK1/2i, SP600125, Calbiochem), GSK3 $\beta$ peptide inhibitor (Calbiochem), or CaM kinase II inhibitor (KN-93, Calbiochem). JNK1/2 inhibition attenuated NFATc3EGFP export to the same extent as the NO donor (fig. 6a). Also, notice that JNK1/2 inhibition increased the plateau of the export to the same level as NO, and that JNK1/2i also decreased the rate of export (fig. 6b). GSK3 and CaM kinase II inhibitors had no effect (data not shown).

To study NFATc3 nuclear import, cells were treated with the chromosomal region maintenance-1 (CRM1) exportin inhibitor, leptomycin B, which blocks nuclear export of NFATc3 and limits normal NFATc3 shuttling in and out of the nucleus. NFATc3-EGFP-transfected cells were incubated with ionomycin in the presence or absence of an NO donor. NO significantly enhanced NFATc3-EGFP nuclear import, as shown in representative images and kinetic curves (fig. 7a and b, respectively). Similarly to what was observed for NFATc3 nuclear export, PKG inhibition abolished NO-enhanced NFATc3 nuclear import and JNK1/2 in- 
Fig. 6. JNK1/2 inhibition reduces NFATc3-EGFP nuclear export. MDCK cells transfected with NFATc3-EGFP plasmid were pretreated with ionomycin $(1 \mu \mathrm{M})$ for $1 \mathrm{~h}$ to induce maximum nuclear accumulation of NFATc 3 , then CsA $(1 \mu \mathrm{M})$ was added (time 0 ) to inhibit further NFATc3 nuclear import; cells were treated with vehicle (control) or with JNK1/2i (SP600125, $80 \mathrm{nM}$ ), and imaged for $30 \mathrm{~min}$. a Kinetic curve of NFATc3-GFP nuclear export. b Kinetic parameters (rate and plateau) of all the treatment groups obtained by fitting the data to a one-phase exponential decay equation. ${ }^{*} \mathrm{p}<0.05$ vs. control; ${ }^{* *} \mathrm{p}<0.05$ vs. NO and JNK1/2i $(\mathrm{n}=4-7)$.
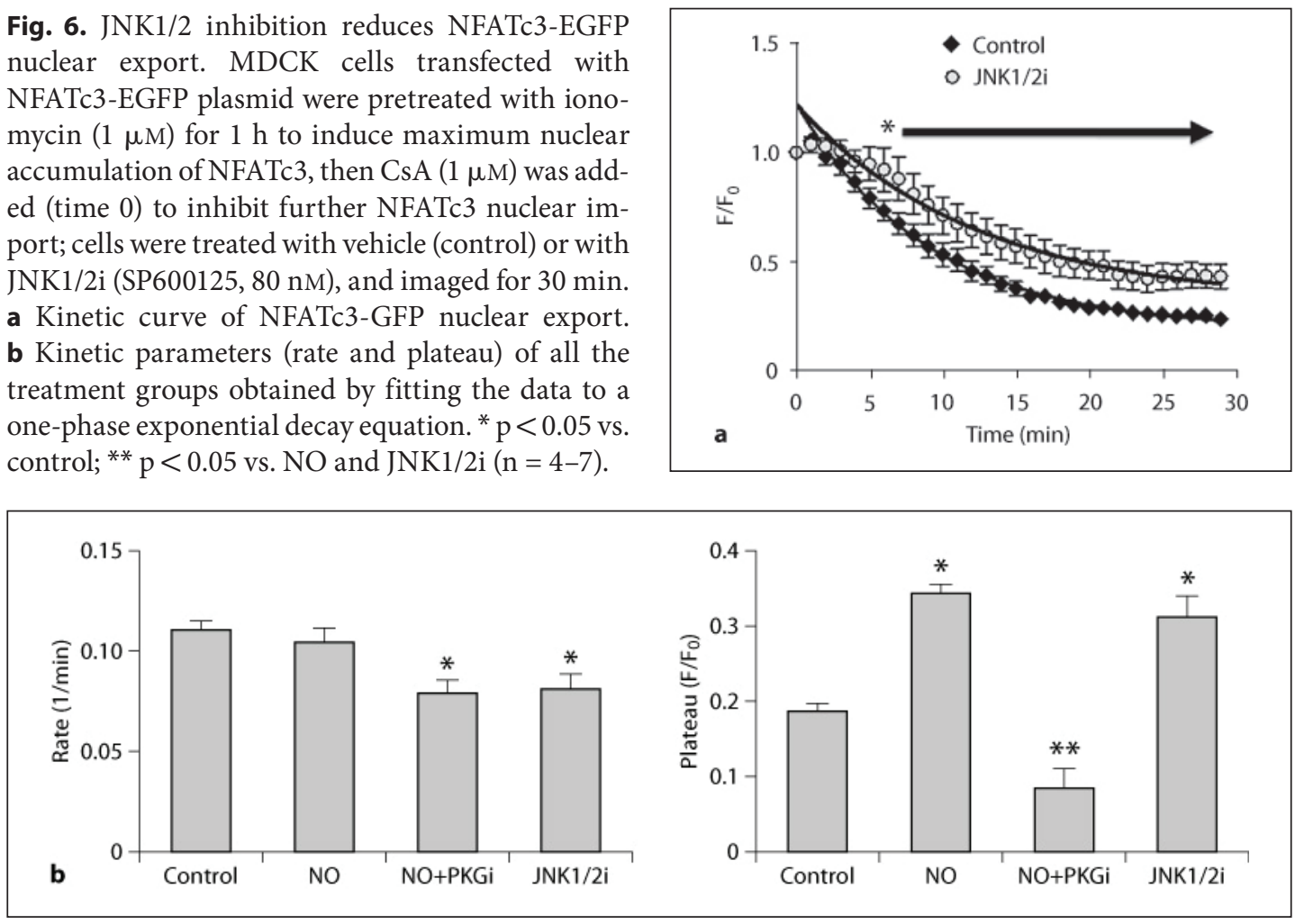

Fig. 7. NO enhances NFATc3EGFP nuclear import. MDCK cells transfected with NFATc3EGFP plasmid were imaged for 30 min under various treatments. Leptomycin B (40 nM) was used to inhibit NFATc3 nuclear export. a Representative images of ionomycin $(1 \mu \mathrm{M})$ and Io+NO donor $(100 \mu \mathrm{M})$-treated cells. b Kinetic curve of NFATc3EGFP nuclear import. ${ }^{*} \mathrm{p}<0.05$ vs. control (leptomycin B alone) and $\mathrm{Io}+\mathrm{NO}+\mathrm{PKGi}$; ${ }^{* *} \mathrm{p}<0.05$ vs. Io+PMA $(n=6)$.

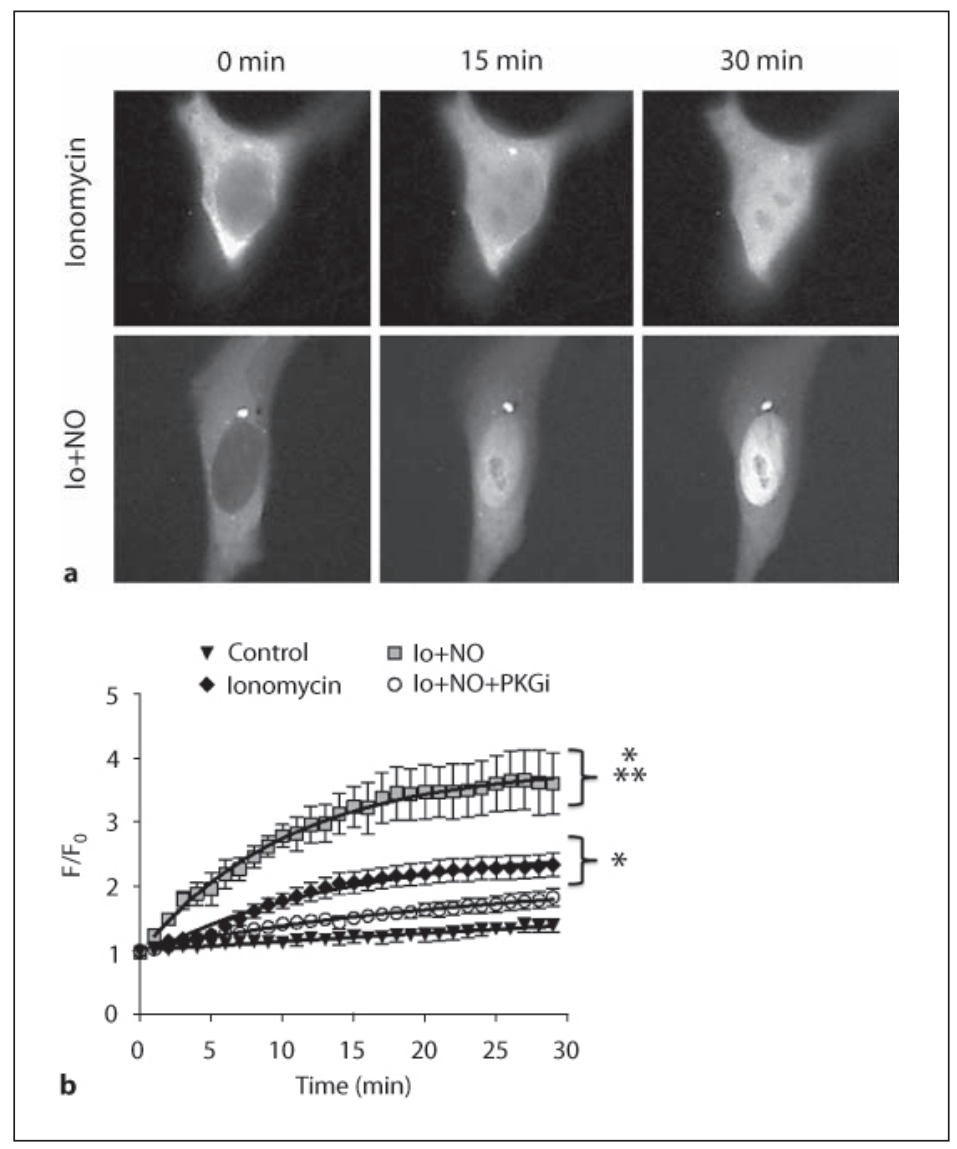


Fig. 8. JNK1/2 inhibition enhances NFATc3-EGFP nuclear import. MDCK cells transfected with NFATc3-EGFP plasmid were imaged for $30 \mathrm{~min}$ under various treatments. Leptomycin B (40 nM) was used to inhibit NFATc3 nuclear export. a Kinetic curve of NFATc3-EGFP nuclear import. * $\mathrm{p}<$ 0.05 from 17 min between groups. b Kinetic parameters (rate and plateau) of all the treatment groups obtained by fitting the data to a one-phase association equation. ${ }^{*} \mathrm{p}<0.05$ vs. ionomycin; ${ }^{* *} \mathrm{p}<0.05$ vs. Io+NO and Io+JNK1/2i $(\mathrm{n}=6)$.
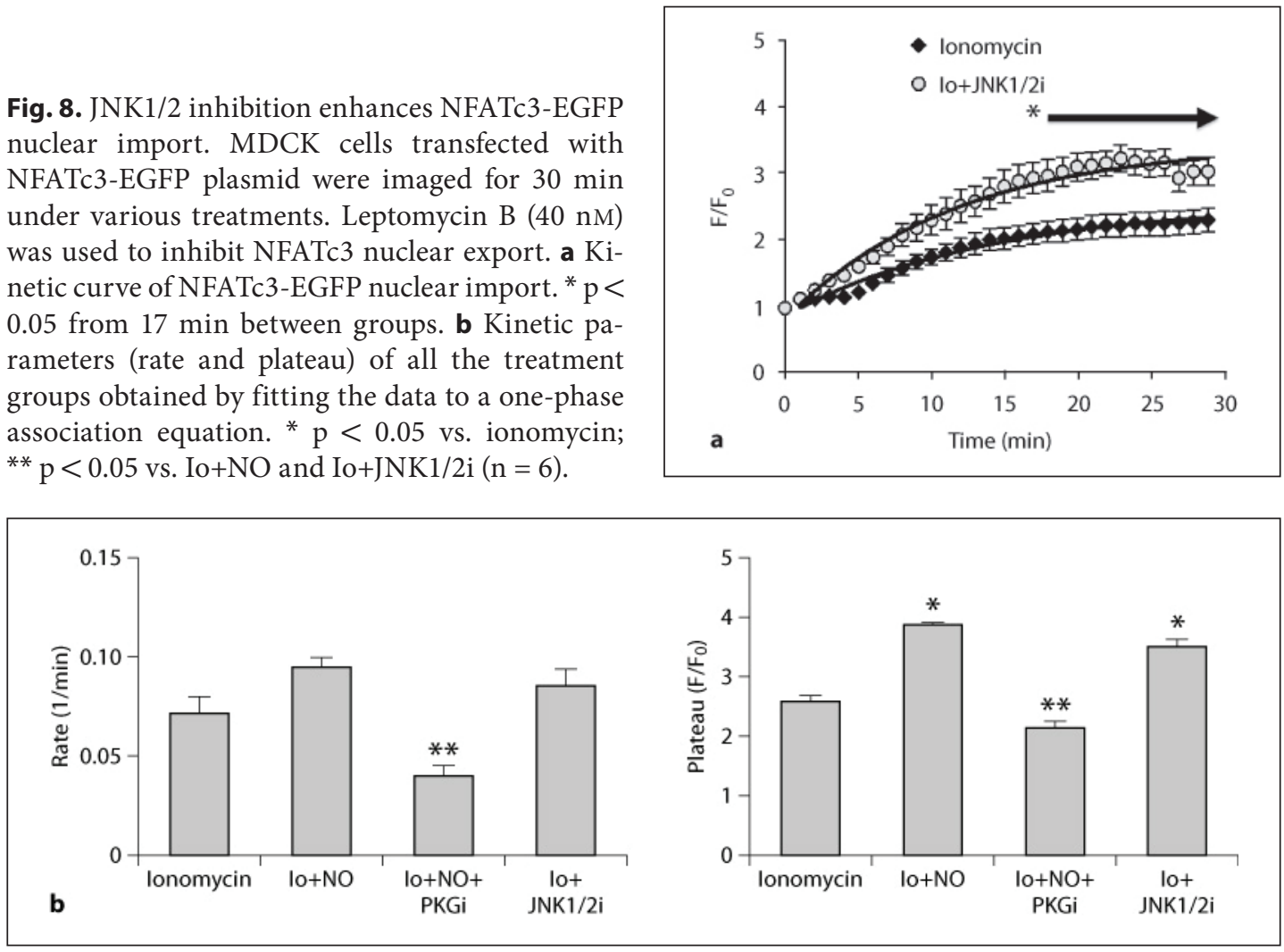

hibition mimicked the effect of NO (fig. 7b, 8a). GSK3 and CaM kinase II inhibitors had no effect (data not shown).

\section{$\mathrm{NO}$ and $\mathrm{Ca}^{2+}$ Inactivate JNK2}

To determine whether $\mathrm{NO}$ and the increase in intracellular $\mathrm{Ca}^{2+}$ lead to inactivation of JNK1 and/or JNK2, the level of phosphorylation of JNK1 and JNK2, an index of JNK activity $[11,19]$, was determined by Western blot in MDCK cells treated with ionomycin, NO and both combined. Our results demonstrate that only the treatment with both (Io+NO) significantly decreased phospho-JNK2 levels (fig. 9). In addition, a strong tendency for a reduction in phospho-JNK1 levels was observed (fig. 9).

\section{Discussion}

$\mathrm{NO}$ is a unique signaling molecule that is implicated in numerous molecular pathways. Our study suggests that NO enhances NFATc-mediated AQP2 expression in mouse renal papilla and demonstrates that it enhances NFATc-mediated AQP2 promoter activity in MDCK cells. Our study supports a novel pathway by which NO might contribute to the regulation of renal water reabsorption.

Elevated water reabsorption can lead to plasma volume increase and hypertension. At the same time, NO is known to be diuretic and natriuretic via sGC/PKG activation [reviewed in ref. 20, 21]; therefore, at the level of the collecting ducts (inner medulla), where the finetuning of urine composition occurs, elevation in NO may enhance NFATc activation, in- 
Fig. 9. $\mathrm{NO}$ and $\mathrm{Ca}^{2+}$ inactivate JNK2. a Representative Western blot of p-JNK1, p-JNK2, and $\beta$-actin in MDCK cells treated for $30 \mathrm{~min}$ with vehicle $(\mathrm{V})$, ionomycin $(1 \mu \mathrm{M})$ and NO donor $(100 \mu \mathrm{M})$. b Summary of the results. ${ }^{*} \mathrm{p}<0.05$ vs. control and ionomycin $(n=6)$.
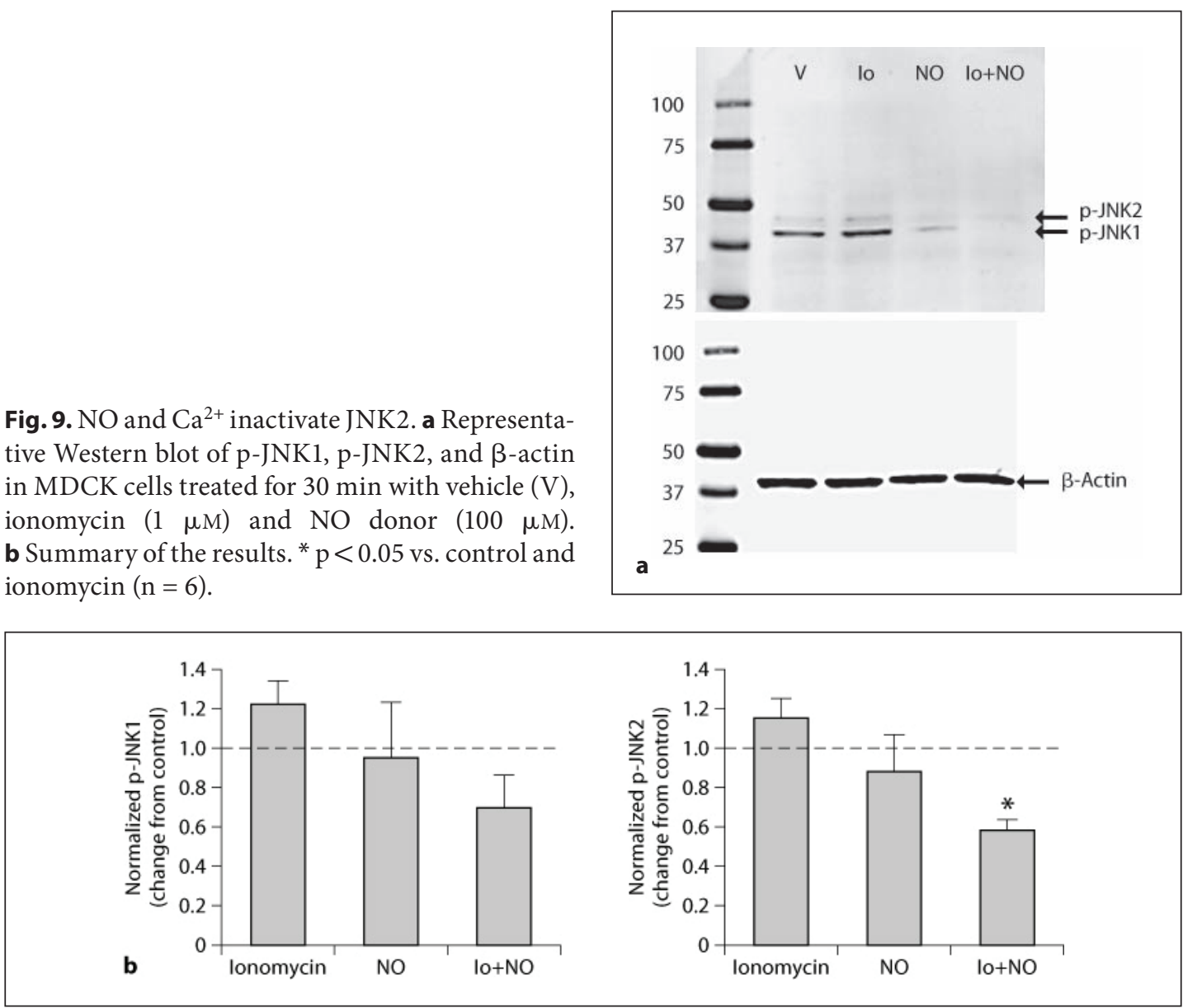

creasing AQP2 expression in order to prevent an excessive loss of water. It is also possible that in certain hypertensive states where renal NO levels are decreased [22], a reduction in AQP2 will compensate for the hypertension [23].

We have previously shown that hypertension induced by chronic inhibition of NOS leads to a down-regulation in outer and inner medulla AQP2 expression [16]. Our current results suggest that NO enhances NFATc-mediated AQP2 expression in isolated mouse renal papilla by regulating AQP2 promoter. Therefore, the reduction in AQP2 expression observed in the rats chronically treated with the NOS inhibitor [16] might not be due to a compensatory response to the elevated arterial pressure, but may be mainly due to the reduced NO levels. Consistent with this possibility, AQP2 expression is blunted in mice lacking all NOS isoforms [24], strongly supporting a role for $\mathrm{NO}$ in regulating AQP2 expression in the renal medulla. Our results also confirm previous findings from Li et al. [15] showing that AQP2 promoter is regulated by the NFATc-AP1 pathway in collecting duct cells.

NO by itself caused a significant, albeit small, increase in AQP2 promoter activity but had no effect on NFATc activity or NFATc3 nuclear import. Similarly, PKC/AP1 activation with PMA increased AQP2 promoter activity but had no effect on NFATc activity (data not shown). These results suggest that NO might also lead to an increase in AP1 activity, which is consistent with previously reported findings in a variety of cell types [25-27]. NFATc activation requires $\mathrm{Ca}^{2+}$-dependent calcineurin-mediated dephosphorylation, explaining the lack of effect of NO by itself on NFATc activity and NFATc3 nuclear import. 
In general, NO signaling involves stimulation of sGC to catalyze the conversion of guanosine triphosphate to cGMP, which in turn activates PKG. However, direct effects of NO have also been described [reviewed in ref. 28]. Our data suggest that $\mathrm{NO}$-enhanced $\mathrm{Ca}^{2+}$ induced NFATc activation is mediated by cGMP. This is supported by the fact that sGC inhibition prevented $\mathrm{NO}$-enhanced $\mathrm{Ca}^{2+}$-induced NFATc reporter activity.

The effect of CsA on the ionomycin- or Io+PMA-induced activity of the NFATc promoter is higher than on the AQP2 promoter. Therefore, we cannot discard the participation of other transcription factors involved in the regulation of the AQP2 promoter activity.

The primary mechanism for NFATc regulation is through control of its cellular localization. Calcineurin, upon activation by $\mathrm{Ca}^{2+}$ and CaM binding, dephosphorylates NFATc, consequently exposing nuclear localization signals allowing for NFATc nuclear translocation. NFATc nuclear accumulation is also controlled by a series of Ser/Thr kinases such as GSK3 $\beta$, CaM kinase II, and JNK1/2 [reviewed in ref. 8, 10]. Under non-stimulated conditions, these kinases keep NFATc in a highly phosphorylated state in the cytosol. After stimulation and once the $\mathrm{Ca}^{2+}$ signal ends, they re-phosphorylate NFATc, inducing its nuclear export by the CRM1 exportin [reviewed in ref. 8, 10].

The enhanced NFATc activity induced by NO could be due to elevated NFATc nuclear import, decreased nuclear export, or both. We found that NO affects both NFATc nuclear import and export. These effects were mediated by PKG, further demonstrating that NOenhanced $\mathrm{Ca}^{2+}$-induced NFATc activation is mediated by sGC/cGMP/PKG, and not by a direct effect of NO.

It has been shown that $\mathrm{NO}$ can nitrosylate and inhibit CRM1, the exportin that transports NFATc out of the nucleus [29]. It is unlikely that NO is decreasing NFATc nuclear export by nitrosylation of CRM1 because the decreased export was prevented by PKG inhibition. Also, it is unlikely that this mechanism is mediating $\mathrm{NO}$-enhanced $\mathrm{Ca}^{2+}$-induced NFATc nuclear import because these experiments were conducted in the presence of the CRM1 exportin inhibitor, leptomycin B.

NO can also inhibit CaM kinase II by nitrosylation, and CaM kinase II has been shown to negatively regulate NFATc in cardiac myocytes [30,31]. However, neither GSK3 $\beta$ nor CaM kinase inhibitors modified NO-enhanced NFATc nuclear import or NO-decreased NFATc nuclear export.

JNK1 has been shown to target NFATc, phosphorylating sites required for NFATc interaction with calcineurin, resulting in a disruption of calcineurin binding and inhibition of NFATc nuclear import [32]. JNK2 also targets NFATc in T cells promoting NFATc export [32]. In addition, we have previously demonstrated that via PKG NO inhibits JNK2, increasing $\mathrm{Ca}^{2+}$-induced NFATc3 nuclear accumulation in vascular smooth muscle [11, 19]. Consistently, we found that an inhibitor of JNK1/2 mimicked the effect of NO on NFATc nuclear export and import, suggesting that NO inhibits JNK1/2. These observations are supported by the results of Western blot showing significantly decreased p-JNK2 levels and a strong tendency to decreased p-JNK1 in cells treated with ionomycin and the NO donor. It has been demonstrated that a reduction in JNK phosphorylation correlates with a reduction in kinase activity $[11,19]$. These results indicate that the already established role of JNK2 and JNK1 in the regulation of NFATc nuclear export and import in T cells and vascular smooth muscle cells, respectively, can be extended to kidney epithelial cells.

The signaling between PKG and the inhibition of JNK1/2 is unknown. PKG has been reported to activate mitogen-activated protein kinase (MAPK) phosphatase, which suppresses the activity of the MAPK cascade $[33,34]$. Because JNK1/2 is a downstream target of the MAPK cascade, it is possible that PKG inactivates JNK1/2 through the activation of MAPK phosphatase. 
In conclusion, this study supports a novel mechanism by which NO could control renal water reabsorption by regulating AQP2 expression via NFATc. In addition, we demonstrate that NO enhances NFATc nuclear import and decreases export via PKG. Then, PKG inhibits JNK1/2 thereby reducing NFATc phosphorylation, and enhancing NFATc nuclear accumulation and transcriptional activity.

\section{Acknowledgments}

This work was supported by a Scientist Development Grant (0535347N) from the American Heart Association to L.V.G.B.

\section{Disclosure Statement}

The authors have no competing interests to declare.

\section{References}

1 Nejsum LN: The renal plumbing system: aquaporin water channels. Cell Mol Life Sci 2005;62:1692-1706.

2 Nielsen S, Frokiaer J, Marples D, Kwon TH, Agre P, Knepper MA: Aquaporins in the kidney: from molecules to medicine. Physiol Rev 2002;82:205-244.

3 Kasono K, Saito T, Saito T, Tamemoto H, Yanagidate C, Uchida S, Kawakami M, Sasaki S, Ishikawa $S$ : Hypertonicity regulates the aquaporin-2 promoter independently of arginine vasopressin. Nephrol Dial Transplant 2005;20:509-515.

4 Lopez-Rodriguez C, Aramburu J, Rakeman AS, Rao A: NFAT5, a constitutively nuclear NFAT protein that does not cooperate with Fos and Jun. Proc Natl Acad Sci USA 1999;96:7214-7219.

5 Miyakawa H, Woo SK, Dahl SC, Handler JS, Kwon HM: Tonicity-responsive enhancer binding protein, a Rel-like protein that stimulates transcription in response to hypertonicity. Proc Natl Acad Sci USA 1999;96:2538-2542.

6 Woo SK, Lee SD, Na KY, Park WK, Kwon HM: TonEBP/NFAT5 stimulates transcription of HSP70 in response to hypertonicity. Mol Cell Biol 2002;22:5753-5760.

7 Hasler U, Jeon US, Kim JA, Mordasini D, Kwon HM, Feraille E, Martin PY: Tonicity-responsive enhancer binding protein is an essential regulator of aquaporin-2 expression in renal collecting duct principal cells. J Am Soc Nephrol 2006;17:1521-1531.

8 Rao A, Luo C, Hogan PG: Transcription factors of the NFAT family: regulation and function. Annu Rev Immunol 1997;15:707-747.

9 Crabtree GR, Olson EN: NFAT signaling: choreographing the social lives of cells. Cell 2002;109:S67-S79.

10 Hill-Eubanks DC, Gomez MF, Stevenson AS, Nelson MT: NFAT regulation in smooth muscle. Trends Cardiovasc Med 2003;13:56-62.

11 Gonzalez Bosc LV, Wilkerson MK, Bradley KN, Eckman DM, Hill-Eubanks DC, Nelson MT: Intraluminal pressure is a stimulus for NFATc3 nuclear accumulation - role of calcium, endotheliumderived nitric oxide, and cGMP-dependent protein kinase. J Biol Chem 2004;279:10702-10709.

12 Gonzalez Bosc LV, Layne J, Nelson MT, Hill-Eubanks DC: Nuclear factor of activated T-cells and serum response factor cooperatively regulate an $\alpha$-actin intronic enhancer. J Biol Chem 2004;280: 26113-26120.

13 De Frutos S, Spangler R, Alo D, Gonzalez Bosc LV: NFATc3 mediates chronic hypoxia-induced pulmonary arterial remodeling with alpha-actin up-regulation. J Biol Chem 2007;282:15081-15089.

14 De Frutos S, Duling L, Alo D, Berry T, Jackson-Weaver O, Walker M, Kanagy N, Gonzalez Bosc L: NFATc3 is required for intermittent hypoxia-induced hypertension. Am J Physiol Heart Circ Physiol 2008;294:H2382-H2390. 


\begin{tabular}{|c|c|}
\hline Nephron Extra 2011;1:124-138 & \\
\hline $\begin{array}{l}\text { DOI: } 10.1159 / 000333066 \\
\text { Published online: October 22, } 2011\end{array}$ & $\begin{array}{l}\text { (c) } 2011 \text { S. Karger AG, Basel } \\
\text { www.karger.com/nne }\end{array}$ \\
\hline
\end{tabular}

15 Li SZ, McDill BW, Kovach PA, Ding L, Go WY, Ho SN, Chen F: Calcineurin-NFATc signaling pathway regulates AQP2 expression in response to calcium signals and osmotic stress. Am J Physiol Cell Physiol 2007;292:C1606-C1616.

16 Albertoni Borghese MF, Majowicz MP, Ortiz MC, Delgado MF, Sterin Speziale NB, Vidal NA: Renal sodium-glucose cotransporter activity and aquaporin-2 expression in rat kidney during chronic nitric oxide synthase inhibition. Nephron Physiol 2007;107:77-86.

17 Livak KJ, Schmittgen TD: Analysis of relative gene expression data using real-time quantitative PCR and the $2^{-\Delta \Delta \mathrm{C}_{\mathrm{T}}}$ Method. Methods 2001;25:402-408.

18 Nomura T, Chang HY, Lu R, Hankin J, Murphy RC, Schuster VL: Prostaglandin signaling in the renal collecting duct: release, reuptake, and oxidation in the same cell. J Biol Chem 2005;280:2842428429.

19 Gomez MF, Gonzalez Bosc LV, Stevenson AS, Wilkerson MK, Hill-Eubanks DC, Nelson MT: Constitutively elevated nuclear export activity opposes $\mathrm{Ca}^{2+}$-dependent NFATc3 nuclear accumulation in vascular smooth muscle: role of JNK2 and Crm-1. J Biol Chem 2003;278:46847-46853.

20 Herrera M, Ortiz PA, Garvin JL: Regulation of thick ascending limb transport: role of nitric oxide. Am J Physiol Renal Physiol 2006;290:F1279-F1284.

21 Ortiz PA, Garvin JL: Role of nitric oxide in the regulation of nephron transport. Am J Physiol Renal Physiol 2002;282:F777-F784.

22 Sigmon DH, Beierwaltes WH: Influence of nitric oxide in the chronic phase of two-kidney, one clip renovascular hypertension. Hypertension 1998;31:649-656.

23 Lee J, Oh Y, Kim SW: Altered renal expression of aquaporin-2 water channels in rats with experimental two-kidney, one clip hypertension. J Korean Med Sci 2001;16:462-466.

24 Morishita T, Tsutsui M, Shimokawa H, Sabanai K, Tasaki H, Suda O, Nakata S, Tanimoto A, Wang KY, Ueta Y, Sasaguri Y, Nakashima Y, Yanagihara N: Nephrogenic diabetes insipidus in mice lacking all nitric oxide synthase isoforms. Proc Natl Acad Sci USA 2005;102:10616-10621.

25 Pilz RB, Suhasini M, Idriss S, Meinkoth JL, Boss GR: Nitric oxide and cGMP analogs activate transcription from AP-1-responsive promoters in mammalian cells. FASEB J 1995;9:552-558.

26 Chen TL, Wu GJ, Hsu CS, Fong TH, Chen RM: Nitrosative stress induces osteoblast apoptosis through downregulating MAPK-mediated NFKB/AP-1 activation and subsequent Bcl-XL expression. Chem Biol Interact 2010;184:359-365.

27 Boggaram V, Chandru H, Gottipati KR, Thakur V, Das A, Berhane K: Transcriptional regulation of SP-B gene expression by nitric oxide in H441 lung epithelial cells. Am J Physiol Lung Cell Mol Physiol 2010;299:L252-L262.

28 Lima B, Forrester MT, Hess DT, Stamler JS: S-Nitrosylation in cardiovascular signaling. Circ Res 2010;106:633-646.

29 Wang P, Liu GH, Wu K, Qu J, Huang B, Zhang X, Zhou X, Gerace L, Chen C: Repression of classical nuclear export by S-nitrosylation of CRM1. J Cell Sci 2009;122:3772-3779.

30 Song T, Hatano N, Kambe T, Miyamoto Y, Ihara H, Yamamoto H, Sugimoto K, Kume K, Yamaguchi F, Tokuda M, Watanabe Y: Nitric oxide-mediated modulation of calcium/calmodulin-dependent protein kinase II. Biochem J 2008;412:223-231.

31 MacDonnell SM, Weisser-Thomas J, Kubo H, Hanscome M, Liu Q, Jaleel N, Berretta R, Chen X, Brown JH, Sabri AK, Molkentin JD, Houser SR: CaMKII negatively regulates calcineurin-NFAT signaling in cardiac myocytes. Circ Res 2009;105:316-325.

32 Chow CW, Rincon M, Cavanagh J, Dickens M, Davis RJ: Nuclear accumulation of NFAT4 opposed by the JNK signal transduction pathway. Science 1997;278:1638-1641.

33 Jacob A, Molkentin JD, Smolenski A, Lohmann SM, Begum N: Insulin inhibits PDGF-directed VSMC migration via NO/cGMP increase of MKP-1 and its inactivation of MAPKs. Am J Physiol Cell Physiol 2002;283:C704-C713.

34 Sun D, Huang A, Recchia FA, Cui Y, Messina EJ, Koller A, Kaley G: Nitric oxide-mediated arteriolar dilation after endothelial deformation. Am J Physiol 2001;280:H714-H721. 